\title{
Epidemiological and Clinical Studies in Patients with Mumps in Pleven District, Bulgaria, 2000-2008
}

\author{
Milena Karcheva and Galya Gancheva \\ Depatment of Infectious Diseases, Epidemiology, Parasitology and Tropical Medicine, Medical University, Pleven, Bulgaria
}

\begin{abstract}
Introduction: The implemented during recent 2-3 decades specific immunoprophylactics against mumps led to serious changes on the intensity and dynamics of the disease all over the world. Objectives: To perform epidemiological analysis of the trend of the disease outbreak for the period 2000 till 2008; to investigate the clinical cases with hospitalized subjects for the same period in Pleven district, Bulgaria. Materials and Methods: There have been used data from the National Centere of Infectious and Parasite Diseases, data reports and analysis of Regional Authority for Protection and Control of Public Health-Pleven. One hundred sixty one patients, suffering mumps, hospitalized in Clinic of Infectious Diseases, were included in the survey. Results: For the period 2000-2008 in Pleven district 974 persons fell ill with mumps. The most inflicted were children and young adults: 10-14 and 15-19 years old $-517(53.08 \%)$ in the first group; $282(28.95 \%)$ in the second age group. There were defined the following clinical forms of mumps infection between the hospitalized persons. Conclusion: The conducted clinical-epidemiological survey of mumps shows that in the conditions of popular specific immunoprophylactics the next epidemiological characteristics undergo changes.
\end{abstract}

Key words: Mumps, morbidity, epidemiological process, clinical forms.

\section{Introduction}

The implemented during recent $2-3$ decades specific immunoprophylactics against mumps led to serious changes on the intensity and dynamics of the disease all over the world.

The popular vaccine coverage on susceptible population in Bulgaria started at the beginning of seventies in the past century. Till the year of 1982 there was applied Bulgarian vaccine, strain Sofia-6. The vaccine range till 1978 increased to $100 \%$ [13]. The outbreak of the disease reduced for about 5 years, but then increased again in $1980-6523$ children have been recorded, 4345 (66.59\%) of them immunized. The highest outbreak of the disease at that dynamics till the eighties was recorded at the age range 4-7 years old patients. After 1981 the most inflicted age is $8-14$ years old. During the recent 1-2 decades the cases shifted to the age of 15-19 years old. Those changes of the

\footnotetext{
Corresponding author: Milena Karcheva, MD, PhD, research fields: epidemiology. E-mail: milena_karcheva@abv.bg.
}

disease are defined indisputably by the dynamics of the popular immunity whose level does not last for a long time and is lacking epidemiological efficiency $[10,14$, 17, 19]. During the 90-ies and the period 2000-2008 in Bulgaria the beginning of immunization by combined vaccine was set (measles, mumps and rubella). Since 1992 there was introduced immunization by three vaccines to 13-months-old children and since 2001, immunization to 12-years-old children. The immunization status has been recorded as improved and the intensity of the epidemiological process reduced although cases have been recorded of immunized with three vaccines as well and such of reimmunized ones $[7,8,18,20]$.

The global analysis of mumps outbreak during the last 1-2 years shows similar trend in other countries as well - England, USA, Croatia, Austria, Spain etc. [1-3].

With this study we aimed to perform epidemiological analysis of the trend of the disease outbreak for the period 2000 till 2008; to investigate 
the clinical cases with hospitalized for the same period in Pleven district, Bulgaria.

\section{Materials and Methods}

There have been used data from the National Centre of Infectious and Parasitic Diseases. Data reports and analyses of Regional Authority for Protection and Control of Public Health - Pleven also have been used to outline the characteristics of the epidemiological process of the district. One hundred sixty one patients were surveyed, suffering mumps, hospitalized in Clinic of Infections at "Dr. Georgi Stranski" Hospital Pleven for the period 2000-2008, emphasizing on their epidemiological anamnesis, age and sex, immunization status, seasonal spread of disease, clinical forms and complications, manifested defined epidemiological conclusions. For the investigation were used epidemiological method, descriptive method, epidemiological observation, and epidemiological analysis. Creating data base of observation, their statistical processing and visualization of results were performed with MS Excel v. 2003. The statistical analysis was conducted through software statistical program Stat graphics Plus Version 2.1.

\section{Results}

On Fig. 1 is shown the trend of the mumps disease outbreak in Pleven district and in Bulgaria. The analysis of the data allows performing comparative assessment of the epidemiological process dynamics of the district and the country. The index of the outbreak in Bulgaria was shifting from 1.2 in 2004 to 73.2 in 2008. The trend of the investigated period was ascending. For the same period in Pleven district a descending trend was being observed. The index of the outbreak was shifting from 114.4 in 2001 to 2.0 in 2008 .

For the period 2000-2008 in Pleven district 974 persons fell ill with mumps, 639 (66\%) being men and 335 (34\%) women. The urban population is more frequently subject to the disease $-700(72 \%)$ in the cities and 274 (28\%) in the villages. Data for the spread of the mumps suffering persons as per age groups is presented in Table 1. As seen in the table cases of mumps are recorded in all age groups. The most inflicted are children and young adults, 10-14 and 15-19-years-old; 517 (53.08\%) in the first group and respectively $282(28.95 \%)$ in the second age group.

The distribution of recorded cases as per months is presented to Fig. 2. The graphic shows that the outbreak is spreading out annually. The greatest number of cases -175 have been recorded in June and 139 in March.

For the period 2000-2008 the Clinic of infectious diseases, University Hospital, Pleven hospitalized 161 persons. They represent $16.5 \%$ of the total registered number of the district. One hundred thirty three (83\%) were men and 28 (17\%) women. The predominating patients were males and the ratio man to women was $5: 1$. The surveyed patients were within the age of 10 months to 42-years-old. $\bar{x}=17.69$; $\mathrm{sd}=6.49$. The distribution as per age groups was the following: 15-19-years-old (40\%), 20-24-years-old (25\%), 10-14-years-old (16\%), 5-9-years-old $\quad(7 \%)$, 25-29-years old (6\%), 30-34-years-old (2\%), 0 -4-years-old (2\%), 35-39-years-old and over $40-$ years-old $-1 \%$. The anamnestic data show that only $10 \%$ of the patients inform for immunization

Table 1 Age groups distribution of mumps in Pleven district for the period 2000-2008. Bolded are the most affected groups.

\begin{tabular}{cc}
\hline $\begin{array}{c}\text { Age groups } \\
\text { (years) }\end{array}$ & $\begin{array}{c}\text { Number of cases (n) } \\
\text { Relative index (\%) }\end{array}$ \\
\hline $0-4$ & $18 / 1.85$ \\
\hline $5-9$ & $44 / 4.52$ \\
\hline $\mathbf{1 0 - 1 4}$ & $\mathbf{5 1 7} / \mathbf{5 3 . 0 8}$ \\
\hline $\mathbf{1 5 - 1 9}$ & $\mathbf{2 8 2} / \mathbf{2 8 . 9 5}$ \\
\hline $20-24$ & $60 / 6.16$ \\
\hline $25-29$ & $19 / 1.95$ \\
\hline $30-34$ & $20 / 2.05$ \\
\hline $35-39$ & $4 / 0.41$ \\
\hline $40-49$ & $8 / 0.82$ \\
\hline over 50 & $2 / 0.21$ \\
\hline
\end{tabular}


against mumps, $11 \%$ of them, not immunized and $79 \%$ without information for vaccination status. The data of the epidemiological anamnesis show that $50 \%$ of surveyed persons do not report for contacts with sick people, suffering mumps, and the remaining $50 \%$ have been in contact with mumps suffering patients within the family or in school.

Based on the clinical data, lab indexes, serologic and other examinations of the hospitalized persons the following clinical forms of mumps infection were defined (Fig. 3) - mumps only is established at 18\% of the patients; combination of two localizations mumps and pancreatitis (36\%), mumps and orchitis (26\%), mumps and meningitis - $(6 \%)$, combination of three-mumps, orchitis and pancreatitis - $(6 \%)$; mumps, orchitis and meningitis - (4\%); mumps, pancreatitis and meningitis - $(2 \%)$. The assessed four localizations are mumps, orchitis, pancreatitis and meningitis - $(2 \%)$.

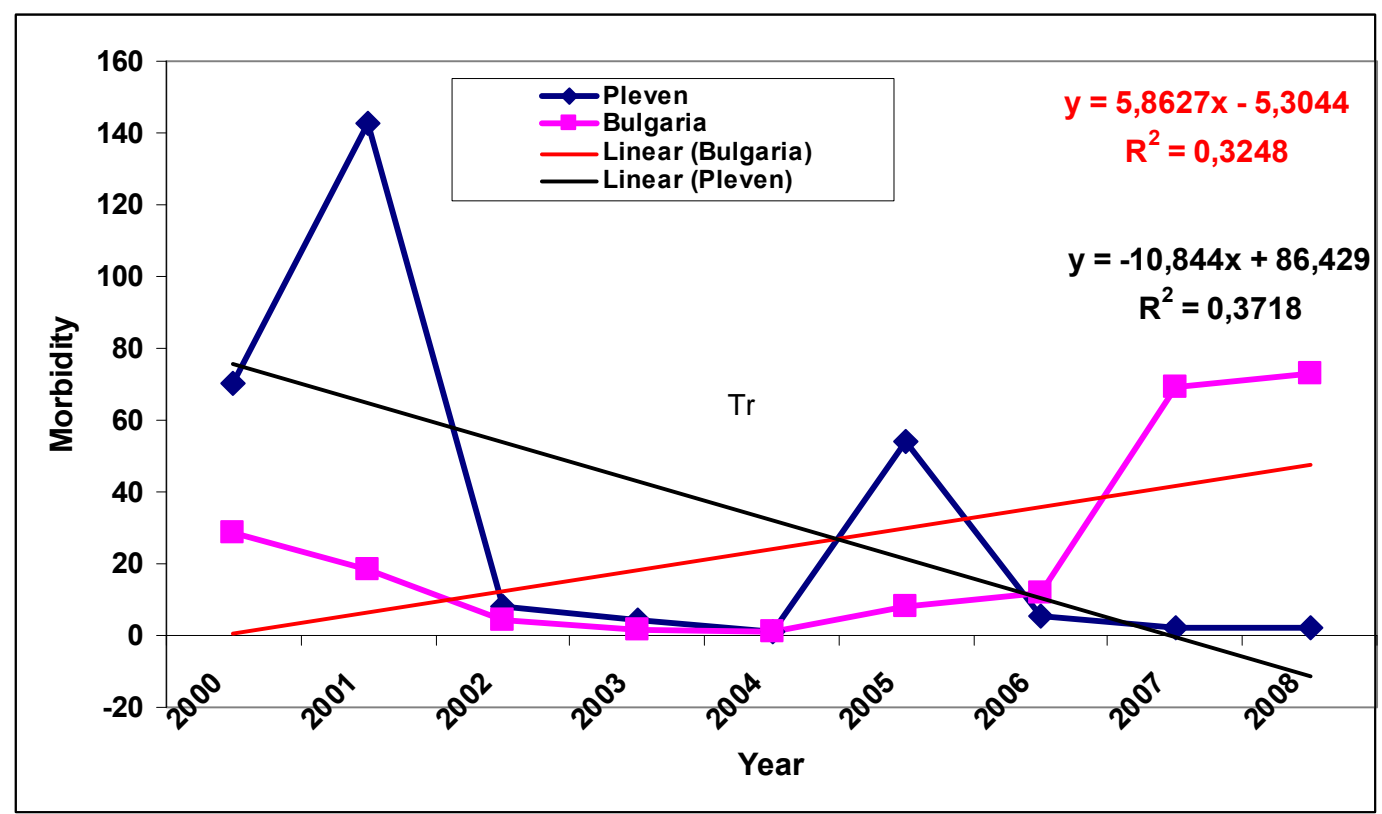

Fig. 1 Trend of the morbidity (for 100000 persons) suffering mumps in Pleven district and Bulgaria for the period 2000-2008.

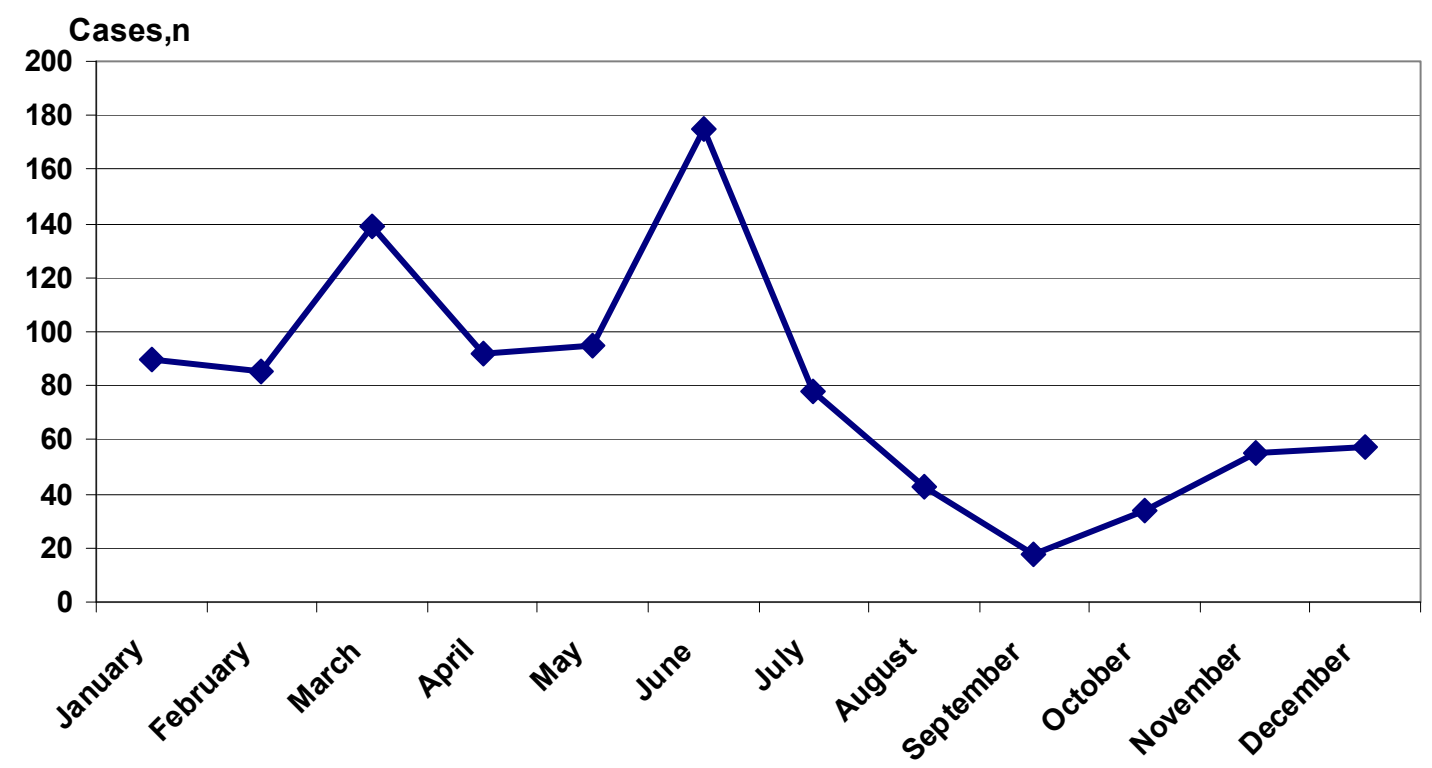

Fig. 2 Monthly distribution of the mumps cases in Pleven district for 2000-2008. 


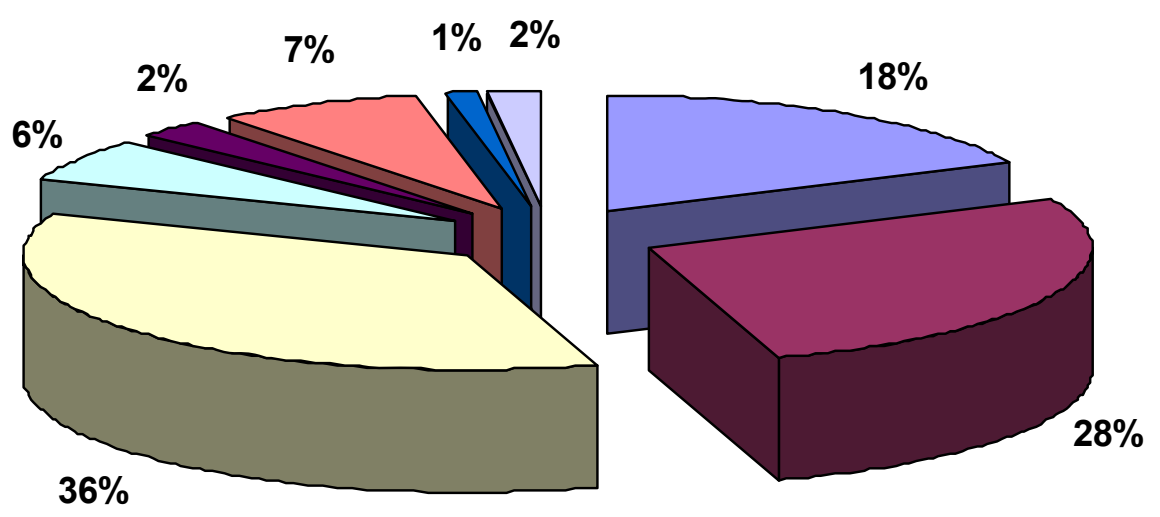

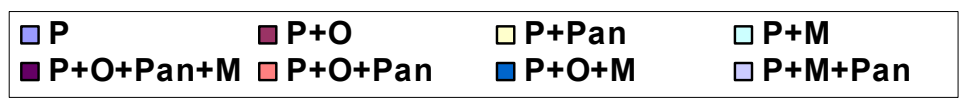

Fig. 3 Clinical forms of mumps infection. ( ${ }^{*} \mathrm{P}$ - parotitis; $\mathrm{Pan}$ - pancreatitis; $\mathrm{O}$ - orchitis; $\mathrm{M}$ - meningitis)

As seen from the data at $82 \%$ of the patients, multiple localization of mumps infection is recorded. The infection with two organs localizations $(70 \%)$ is predominating. The most frequently met combination is parotitis and pancreatitis (36\%), followed by parotitis and orchitis (28\%). The data show that at all patients infliction is noted of the parotitis glands $(100 \%)$. Moderate-heavy course of the disease is predominating - over $90 \%$ of the cases. The course of the disease went benignly. Lethal outcome have not been recorded.

\section{Discussion}

Upon the implementation of popular specific immunoprophylactics in Bulgaria the outbreak of the mumps decreased. The investigated period includes years when Bulgaria introduced two staged immunization scheme of the vulnerable population with three vaccines (MMR). On the background of the insignificant outbreak in $2004-1.2$ to 100000 at the end of the period an up going trend is being observed. That was the result of the occurred epidemic outbreaks in the separate regions of the country. Such outbreaks have been noted in other countries with mandatory immunizations - Britain, Ireland, Austria, Spain, USA, Canada etc. [4-6]. The data show that during the recent years the outbreak of the mumps in Bulgaria does not differ substantially to the situation in other countries that introduced the two staged immunization scheme, actually registration of the outbreaks, concerning young adults.

Pleven district is one of the inflicted by epidemic outbreaks and during the investigated period two epidemic outbreaks have been noted - in 2001 with the index of 142.47 and in 2005-54.11. An interim epidemic period of 3 years has been noted, characterized by lower morbidity. That preserved cycles in the course of the epidemic process is characteristic, being observed and prior of the immunization period. The trend in the district is said to be decreasing which demonstrates that the vulnerable population also reduced in number due to the performance of immunizations and/or disease.

The given season is another specific characteristic of the infectious diseases. The spread of the disease with mumps commences in the autumn and last maximum during spring. For the period 2000-2008 in the district we investigated we recorded annually mumps. The 
number of cases increased during the months of March and June. The increase of the cases in spring and summer shows that in the conditions of popular specific immunoprophylactics the characteristics for winter-spring mumps is not to be noted.

Despite we accept equal vulnerability of both sexes to the infection during the recent decades the clinically forms of the disease are predominately recorded with male patients as seen from our survey (ration 2:1 in favour of males from all registered cases in the district and 5:1 with the hospitalized). Similar distribution as per sex is not reported in the surveys of other authors. Greater outbreak with the urban population is related to the higher rate of density in cities and towns.

The age distribution of the patients is important feature of the infectious diseases. The most affected groups with registered mumps in the district were those of 10-14-years-old-517 (53.08\%) and 15-19-years-old - 282 (28.95\%). The data correspond to the age distribution of the country for the referenced period. The implementation of the two-staged application of the vaccine shifted the outbreak of mumps to the groups 15-19-years-old and 20-29-years-old subjects. In a number of countries higher outbreak was being registered in older age groups [10].

In the hospitalization subjects the biggest groups were the patients 15-19-years-old (40\%) followed by 20-24-years-old (25\%), 10-14 years old (16\%). These data can be explained by the fact that the hospitalized patients with more than one organic localization and in no less percent (about 30\%) of them have parotitis and orchitis. The latter occurs more frequently with young adults after the puberty $[15,18]$.

It is interested from practical point of view the issue of the multiplicity of organic localizations at mumps infection [2]. The combination of two-three localizations is comparatively frequent event. More of three localizations is an exception to be met in the literature, so that investigation is presenting great interest. All cases of parotitis with extra salivary localization is a reason for hospitalization of the persons $[12,16]$. Multiple localization of the infection correlates with the gravity in the course of the infectious process. We noted extended hospital stay with patients - up to 25 days when conducted the investigation. The infliction on the pancreas is the leading extra salivary localization at the surveyed by us patients, suffering parotitis. There was diagnosed parotitis and pancreatitis with $36 \%$ of the hospitalized. The infliction on the pancreas occurs at three or four localizations of the infection, thus the percent of pancreas infliction reaching $48 \%$. That high percent of the pancreas occurrence at mumps infection differs from the information, provided in the literature. There are various data on the pancreatitis frequency at parotitis infection, between $5 \%$ and $20 \%$. Most of the authors accept that pancreatitis is a rare case to be met. Heavy cases of pancreatitis without parotitis in children have been described extremely rarely. For the period 2000-2008 at 38\% of the hospitalized male persons, orchitis has been diagnosed. The following combinations of orchitis have been observed: parotitis and orchitis at $28 \%$, parotitis, orchitis and pancreatitis at $7 \%$, parotitis, orchitis and meningitis at $1 \%$ and parotitis, orchitis, pancreatitis and meningitis with four $(2 \%)$. The clinical manifestations of the investigated patients in our survey do not differ from those reported in literature $[9,11,12,16]$. Cases of isolated orchitis have been reported, which is the sole clinical manifestation of the parotitis infection but not yet established to our patients.

The serous meningitis is one of the substantial localizations of the mumps infections. For the period 2000-2008 in the clinic of infectious diseases 21 patients with parotitis meningitis have been admitted. They represent $13.04 \%$ of all hospitalized for the same period. The distribution as per sex is the following: males - 19 (90.48\%), females - 2 (9.52\%). The observations show that males suffer more frequently with meningitis, which has been established and confirmed by us. 


\section{Conclusion}

The conducted clinical-epidemiological survey of mumps shows that in the conditions of popular specific immunoprophylactics some epidemiological characteristics undergo changes: the immunoprophylactics reduces the intensity of the epidemic process; the outbreak shifts to older age groups; seasonal features does not occur; vaccine takes the role of process regulation, which makes it the most important means to fight the disease. Regarding the clinical course of the disease at all clinical forms it is noted impact on salivary glands, various organs localizations of the mumps infections, being registered. The most frequent extra salivary localization is the parotitic pancreatitis, followed by parotitic orchitis. The course of the disease is smooth and benign.

\section{References}

[1] H. Bernard, N. G. Schwarz, A. Melnic, V. Bucov, N. Caterinciuc and R. G. Pebody et al., Mumps outbreak ongoing since October 2007 in the Republic of Moldova, Euro Surveillance 13 (1-3) (2008) 102-104.

[2] J. A. Boga, M. de Oňa, A. Fernandes-Verdugo, D. Gonzalez, A. Morilla and M. Arias et al., Molecular identification of two genotypes of mumps virus causing two regional outbreaks in Asturias, Spain, Journal of Clinical Virology 42 (4) (2008) 425-428.

[3] N. Boxall, M. Kubinyiova, V. Prikazsky, C. Beneš and J. Castkova, An increase in the number of mumps cases in the Czech Republic, 2005-2006, Eurosurveillance 13 (4-6) (2008) 1-4.

[4] BMJ USA, Mumps outbreak among young adults in UK, BMJ 331 (2005) E363-E364.

[5] P. A. Briss, L. J. Fehrs, R. A. Parker, P. F. Wright, E. C. Sannella and R. H. Hutcheson et al., Sustained transmission of mumps in a highly vaccinated population: Assessment of primary vaccine failure and vaccine induced immunity, J Infect Dis. 169 (1) (1994) 77-82.

[6] Caralee E. Caplan, Mumps in the era of vaccines, Canadian Medical Association Journal 160 (6) (1999) 865.

[7] J. Castilla, M. Garcia Genoz, M. Arriazu, M. Fernandez-Alonso, V. Martinez-Artola and J. Etxeberria et al., Effectivness of Jeryl Lynn-containing vaccine in Spanish children, Vaccine 27 (15) (2009) 2089-2093.

[8] M. M. Cortese, H. T. Jordan, A. T. Curns, P. A. Quinlan, K. A. Ens and P. M. Denning et al., Mumps vaccine performance among university students during a mumps outbreak, Clin Infect Dis. 46 (8) (2008)1172-1180.

[9] C. Cohen, J. M. White, E. J. Savage, J. R. Glynn, Y. Choi and N. Andrews et al., Vaccine effectiveness estimates, 2004-2005 mumps outbreak, England, Emerg Infect Dis. 13 (1) (2007) 12-17.

[10] G. H. Dayan and S. Rubin, Mumps outbreaks in vaccinated populations: Are available mumps vaccines effective enough to prevent outbreaks? Clin Infect Dis. 47 (11) (2008) 1458-1467.

[11] I. Dikov, G. Popov, A. Apostolova, D. Dimitrova, D. Stoinova and K. Plochev et al., Multiple and rare localizations and clinical manifestations at mumps infection, Sofia, Bulgarian Journal of Medicine 2 (1) (2008) 65-67.

[12] P. Ilieva, M. Atanasova, T. S. Doichinova, I. Simova and H. Tsvetanova, Clinical manifestations of mumps infection, Sofia, Infectology (3) (32) (2000) 16-18.

[13] S. O. Gee, D. Flanagan, M. Fitzgerald, S. Cotter, Mumps in Ireland, 2004-2008, Eurosurveillance 13 (4-6) (2008) $1-3$.

[14] R. K. Gupta, J. Best and E. MacMahon, Mumps and the UK epidemic 2005, BMJ 330 (2005) 1132-1135.

[15] H. Jalal, G. Bahadur, W. Knowles and L. Jin Brink, Mumps epididymo-orchitis with prolonged detection of virus in semen and the development of anti-sperm antibodies, J. Med. Virol. 73 (1) (2004) 147-150.

[16] M. Karcheva, P. Hristova, S. Kozarova and B. Bonev, Retrospective investigation of the epidemiological process of epidemic mumps in Pleven district for five years period, Trakia Journal of Sciences 6 (2) (Suppl. 3) (2008) 147-150.

[17] M. Kojouharova, A. Kurchatova, L. Marinova and T. Georgieva, Mumps outbreak in Bulgaria, 2007: A preliminary report, Euro Surveill.12 (12) (2007) 3162, available online at: http://www.eurosurveillance.org /ViewArticle.aspx?ArticleId=3162.

[18] J. C. Lopez Pacios, L. Parra Muntaner, M. C. Piňeiro Fernandez, S. C. Gomez Cisneros, E. Sanchez Sanchez and J. A. Rivas Escudero et al., Mumps orchitis: Review of 8 cases, Arch Esp Urol. 51 (4) (1998) 331-333.

[19] D. G. Mackenzie, G. Craig, N. F. Hallam, J. Moore, J. Stevenson, Mumps in a boarding school: description of an outbreak and control measures, Br. J. Gen. Pract. 56 (528) (2006) 526-529.

[20] D. W. Park, M. H. Nam, J. Y. Kim, J. W. Sohn, Y. Cho and K. J. Song et al., Mumps outbreak in a highly vaccinated school population: Assessment of secondary vaccine failure using IgG avidity measurements, Vaccine Apr 24, 2007, PMID: 17498856. 